\title{
Deep vein thrombosis
}

\author{
G. E. MAVoR \\ M.A., M.D., Ch.M., F.R.C.S.E. \\ Aberdeen Royal Infirmary
}

Although the cause of deep vein thrombosis remains uncertain Virchow's triad of vessel wall disease, change in the composition of the blood and slowing of the blood stream remains generally acceptable as covering most of the aetiological possibilities. The evidence also supports the view that venous stasis is important. Venous return from the lower limb is facilitated by elevation of the leg, exercise, especially of the calf muscles, and deep breathing. Venous circulation through the main veins of the lower limb is slowed when patients are supine, and the legs horizontal and motionless (McLachlin et al., 1960). Observations on pressures recorded in the inferior vena cava also illustrate the beneficial effect of an active calf muscle pump on venous return (Lancet, 1962). General clinical observations support these findings. For instance it is well established that the longer the period in bed the greater the incidence of thrombo-embolism, and this particularly so in the elderly (Gibbs, 1957; Sevitt, 1959).

Local factors also play a role in venous stasis and thrombosis. Careful post-mortem studies have shown that thrombi tend to form at valve pockets, vein saccules, and at vein junctions, and in areas where venous compression from neighbouring structure occurs (Gibbs, 1957; Sevitt, 1959). Into the latter category comes the left common iliac vein. This has not only a transverse lie and entry into the inferior vena cava, owing to developmental factors, but may be compressed by the overlying right common iliac artery. Hence there is a ready explanation for the higher incidence of proximal or iliofemoral venous thrombosis on the left side as compared with the right. Patients with myocardial infarction, with acute obliterative peripheral arterial disease, with major amputations, and those with femoral neck fractures or operations on the hip joint all represent high risk groups, in which a major aetiological factor may be venous stasis. It seems clear too that venous stasis is not only a factor in the production of primary thrombosis, but is also important in the complete occlusion of the deep vein and in the extension of the thrombus once formed.

As specific factors in aetiology remain unknown, so the problems of management in large measure remain unsolved. To a considerable extent these problems have been obscured for more than a quarter of a century by the illusion of safety created by the anticoagulant drugs. The erroneous idea that these drugs were effective in all forms of established venous thrombo-embolism has delayed the proper correlation of clinical and pathological events, without an understanding of which treatment is unlikely to be either meaningful or successful.

Regarding their therapeutic role, it should now be more widely recognized that there is no proof of the effectiveness of the anticoagulant drugs in this disease. Furthermore, their use in treatment is not without risk. The limitations of such therapy are due to many factors, not least the consideration that fibrin is only one of the components of a thrombus. The coumarin derivates, acting on coagulation Factors IX, X, II and VII, depress to some extent both the extrinsic and intrinsic thrombo-plastic systems, while heparin acts against Factor IIa (or thrombin). There are, however, many other aspects of the coagulation cascade unaffected by these drugs. It also requires to be stressed that dosage control particularly in regard to the oral coumarin drugs is difficult, and critical evaluation shows that it is impossible to maintain patents all the time on what is recognized as a 'therapeutic level'.

As regards the use of the anticoagulant drugs in prophylaxis, a reasonable clinical evaluation has been carried out and statistical evidence suggests that they are of value (Sevitt \& Gallagher, 1959). However, this does not mean that the problem of venous thrombo-embolism has been eliminated, nor does it mean that protection has been conferred on the individual patient. The effectiveness of these drugs will depend on how early they are given in the course of the patient's hospital stay, how long they are continued, and possibly most important of all, how adequate is their control. However, it is not possible to submit all patients to such treatment, and accordingly anticoagulants have been restricted to patients considered particularly susceptible for one reason or another to venous thrombosis. Even in such circumstances, however, the disease is by no means controlled.

Also in the field of drug therapy, arvin, an 
anticoagulant agent from the Malayan pit viper, and thrombolytic agents such as urokinase and streptokinase are presently under investigation (Mavor \& Galloway, 1970. Arvin has a specific coagulant action on fibrinogen, converting it to an unstable fibrin, which is then lysed so that fibrinogen disappears from the blood. Arvin has also an effect on platelet aggregation, probably mediated through fibrin degradation products.

Studies of variations in platelet behaviour represented by adherence to glass in vitro (Hellem, 1960) and to damaged endothelium (Alfors et al., 1968) have raised the hope that in such changes, where they exist, may lie at least some of the answers to explain the early beginnings of clinical venous thrombosis. Hence the investigation of various drugs affecting platelet behaviour, such as the pyrimidopyrimidine compounds and aspirin, now being undertaken (Bennett et al., 1966; Browse, 1968). 'Macrodex' (dextran of average molecular weight 70,000 ) is probably the most important of these drugs. It is likely that if such drugs are to prove useful it will be in prophylaxis. Dextran 70 not only suppresses platelet activity, but by expanding the plasma volume increases both the cardiac output and venous blood flow. No doubt all these factors play some role in its established beneficial effect in the prophylaxis of deep vein thrombosis. It has the advantage over the oral anticoagulants of being safer, more easily controlled, and more effective. But like them, it does not give individual protection (Lambie et al., 1969).

Prophylactic active regimes directed against venous stasis are common practice, but reports about the benefits of such regimes are conflicting (Allen, 1946; Blodgett \& Beattie, 1946). Confusion arises largely because of the different diagnostic standards used, particularly in early cases, the difficulty of obtaining control series, and the infrequency of the condition. The value of prophylactic measures are difficult to assess against a natural incidence of less than 5\% (Hobsley \& Patey, 1962).

It is important that in the evaluation of various prophylactic regimes, and particularly of drugs, in the field of venous thrombosis, objective methods of assessment are used. In this context a great advance has been the introduction of ${ }^{125} \mathrm{I}$-labelled fibrinogen, by means of which precise information of thrombus formation in the peripheral segment can be obtained (Flanc, Kakkar \& Clarke, 1968). It is to be hoped that with such technique available for objective control, the misleading conclusions, based on clinical assessment, reached in regard to the anticoagulant drugs, will not be repeated.

\section{Clinical classification of deep vein thrombosis}

Mavor \& Galloway (1969a) and Galloway (1969) have suggested a segmental approach in classifyiñ deep vein thrombosis into peripheral or distal (lowefr segment) on the one hand and high or iliofemorat (upper segment) on the other. On pathological, clinical and therapeutic grounds this offers mary advantages, not least the clarification in the clipician's mind that peripheral thrombosis is seldom itself serious, while iliofemoral venous involveme $\overline{\bar{n} \text { t }}$ can be and usually is complicated by acute and chronic morbidity often of severe degree (Mavor Galloway, 1967b, 1969a). The profunda femoris termination (about 2 in. below the groin) is the most convenient site for division of the deep vein into peripheral and high segments.

\section{Natural history of deep vein thrombosis}

For many years thrombosis of the calf veins has been considered to be an event of primary importance in venous thrombo-embolism, and present-dey clinicians continue to place particular stress on the prevention, diagnosis and management of this condition. The prevailing belief is that from of initial site in the calf venous plexuses, by extension in continuity the posterior tibial, popliteal and femoral veins are involved in clotting, the iliac veiñs also being affected on less frequent occasions. Th understanding of the natural history requires to be challenged, for not only are its origins obscure and uncertain, but the evidence accumulated from rese git studies is contradictory.

It has been known for a long time that thrombess formation in the calf veins is a common event. Frof a study of four cases of calf vein thrombosis suffering pulmonary embolism Homans (1934) suggested the्pt extension occurred into popliteal and femoral veins. Bauer (1940) also produced a series of phlebograng of one patient showing obliteration of the femoral vein from progression of a calf vein thrombos Neither Homans nor Bauer reported on the coğ: dition of the iliofemoral segment in these or other cases under investigation. It became understood that in nearly all instances where proximal involvemegt occurred, this was the outcome of initial mobe peripheral thrombosis.

Long before this idea became popular, howev, attention had been drawn to the importance of the iliofemoral segment as an initial site of thrombosis McMurrich (1908) and Ehrich \& Krumbhaar (19632) drew attention to abnormalities about the ilio-cavis orifices and their possible association with the high incidence of thrombosis in that region. Aschorf (1924) also noted this and suggested that large fatal emboli could only come from veins of this size. Mome recently McLachlin \& Paterson (1951), Gibbs (1957) and Sevitt (1959) have stressed the importance of the iliofemoral segments and have shown that $\overrightarrow{\mathrm{g}}$ patients dying of venous thrombo-embolism it wass 
a common finding to have clot forming in different situations in the deep veins at the same time. Of these, the calf veins, the femoro-popliteal segments, the common femoral veins and the common iliac veins were often involved. Several instances with continuous thrombus formation from the popliteal veins as far as the adductor hiatus were observed, but in a total of 420 dissections, neither Sevitt nor Gibbs was able to confirm a single instance where clot had extended in continuity from the peripheral into the iliofemoral system. Clinical experience from venous thrombectomy exactly confirms these pathological findings. In a series of 257 cases of iliofemoral thrombosis treated by thrombectomy, in only twenty-four instances was it considered that intravenous clotting had extended in continuity from the periphery to involve the iliofemoral segment (Mavor \& Galloway, 1969a). The usual finding at venous thrombectomy was of old firm clot in the common iliac vein and fresh clot occluding the external iliac and common femoral. Distal to the profunda termination there might be no thrombus, or if occlusion did exist the clot was fresh with small scattered fragments of old thrombus in it (Mavor \& Galloway, 1969a). Furthermore, it has also been well demonstrated that when pulmonary embolism is the presenting symptom and the legs clinically 'silent', subsequent events showed that in two-thirds of all cases, the site of clot formation was in the iliofemoral segment (Mavor \& Galloway, 1967a).

The internal iliac system usually escapes involvement in this disease. Aschoff (1924) was the first to stress this, also noting that embolism was nto common from these veins. Extensive experience with iliofemoral phlebography either at venous thrombectomy or during investigation confirms these views, as except in rare instances normal internal iliac systems are visualized (Mavor \& Galloway, 1969a).

Involvement of the iliofemoral segment as an initial site in many cases of deep vein thrombosis also explains how sudden venous occlusion of the deep system may occur with acute venous insufficiency affecting the whole leg. In such cases retrograde extension of clotting is to be expected when high occlusion occurs. It is more difficult to accept that clotting will more readily occur in a proximal direction to involve the iliofemoral segment when there is a high venous inflow at the groin via the collateral veins to the peripheral segment which include the profunda femoris and venae comitantes (Mavor \& Galloway, 1969a). It is also known that in $34 \%$ of all legs there is no large direct popliteal-femoral venous link (Mavor \& Galloway, 1967b).

In this context therefore it is inaccurate to regard iliofemoral venous thrombosis as a late example of the disease of venous thrombo-embolism and the inevitable outcome of progressive clotting more distally. Nevertheless, this is the common teaching today. Iliofemoral venous thrombosis is not so common as peripheral involvement, but it must be recognized that it often occurs as the presenting pathological event, that it may be non-occlusive for a considerable time, and that it may give rise to secondary thrombosis by retrograde propagation.

In clinical management much greater emphasis requires to be placed on the iliofemoral segment, and the occurrence of calf or peripheral involvement, whether diagnosed clinically or by other means, should serve only to direct the clinician's attention to the possible simultaneous involvement of the iliofemoral segment, as he should be aware that in this situation venous thrombosis is much more insidious, much more serious and much more damaging in its consequence.

The variable clinical course of deep vein thrombosis is well known. In the past this has presented difficulties in estimating the effect of various methods of treatment. Commonly, peripheral deep vein thrombosis is a fairly bland disease, usually shortlived, self-limiting, and infrequently complicated. Only occasionally does recurrent embolism become a problem in these cases. In iliofemoral disease there is considerable variation in the activity of the disease process. In many instances the disease may be low grade and management presents no great difficulties, but there is a minority of cases where the disease is excessively acute and active. Symptoms and signs are marked, fever and erythrocyte sedimentation rate much raised, the course protracted, and severe complications like caval thrombosis, mesenteric venous thrombosis and venous gangrene more common. Both iliofemoral segments can be involved, either simultaneously or successively, and the superficial veins are often intensely phlebitic. Repeated pulmonary embolism is a common accompaniment, recurrence over the years usual, and the morbidity and mortality high. Whether there is anything to be gained by labelling such cases 'thrombophlebitis migrans' or by associating them with pancreatic disease in particular, or malignant disease in general is doubtful. In most there is no underlying primary pathology. The incidence of this type of severe disease is about 8\% (Mavor \& Galloway, 1969a).

\section{Peripheral (lower segment) venous thrombosis}

It is widely accepted that calf vein thrombosis is a common event. Detailed dissection studies of Frykholm (1940), Murley (1950) and Cotton \& Clark (1965) provide ample justification of this. Evaluation of intravenous clotting by the ${ }^{125} \mathrm{I}-$ labelled fibrinogen gives further confirmation of this, clot formation occurring in this site in probably more than $30 \%$ of all postoperative cases (Kakkar 
et al., 1969). Many of these cases are subclinical. What is not known is how often distal peripheral involvement becomes clinically significant by giving rise to extension in continuity to the iliofemoral segment, or by producing recurrent emboli. The evidence suggests that peripheral and particularly calf vein thrombosis is not in itself of serious clinical importance. Extension to the iliofemoral segment is rare, and in most cases of iliofemoral occlusion, scanning with ${ }^{125}$ I-labelled fibrinogen shows no distal involvement (Mavor \& Galloway, 1970b). With peripheral segment thrombosis adequate collatcral arrangements exist (Fig. 1), acute venous insufficiency does not occur and late morbidity is related to perforator damage, resulting in one form

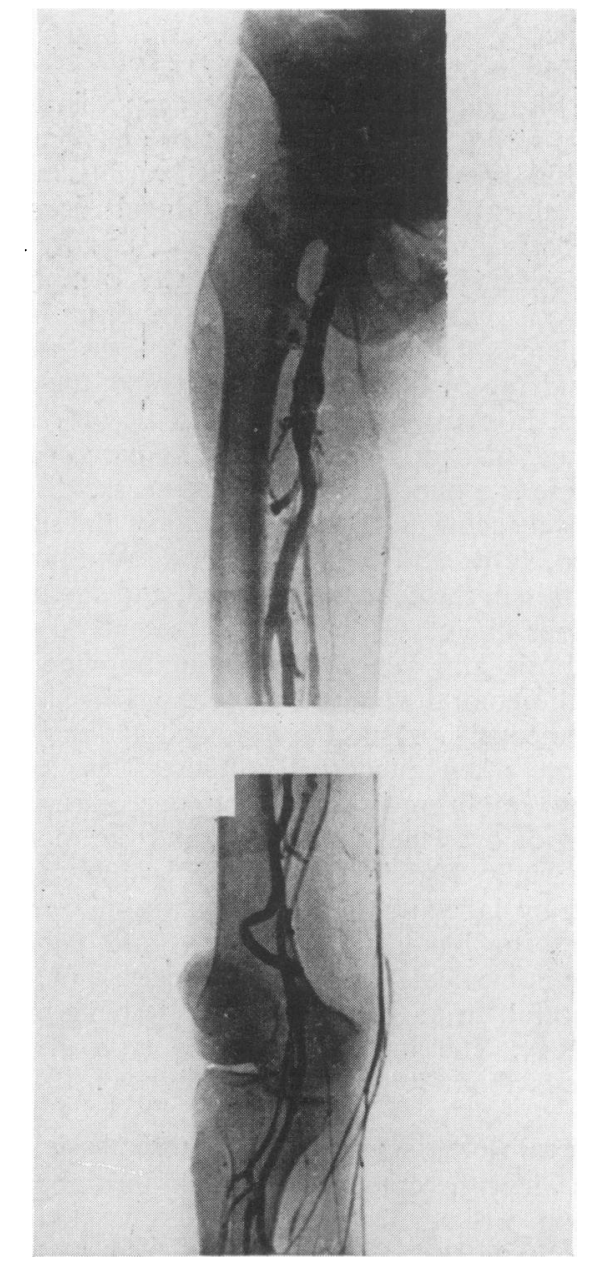

FIG. 1. Complete occlusion of the peripheral venous segment is present extending up to but not beyond the profunda femoris termination. Typical by-passing channels are the profunda femoris vein draining the popliteal area, the venae comitantes, and the less important long saphenous vein. of the postphlebitic syndrome (Cockett \& Jones 1953; Cotton \& Clark, 1965; Mavor \& Gallowağ, 1967b). Pulmonary embolism with its origin in this segment is uncommon and not usually significant, cannot be massive and can only become serious because of the cumulative effect of many sm平 emboli leading to intcrmittent pyrexia, unusual che radiological signs, pulmonary hypertension and right heart failure. In a series of 237 cases of pu monary embolism, in only 33 or $13 \%$ was the peri⿻ pheral segment the site for recurrent embolism. There were no fatalities in this group (Mavor. Galloway \& Karmody, 1970a). However, before the्ट peripheral segment can be incriminated as a soure of pulmonary embolism, even if the clinical diag. nosis is confirmed by phlebography and periphera् scanning using ${ }^{125} \mathrm{I}$-labelled fibrinogen, objective evidence is required that the iliofemoral segments do not contain clot. This is most easily obtained by phlebography (Mavor, Halloway \& Mahaffy, 196964

The diagnosis of peripheral deep vein thrombosis has recently been facilitated by the development of peripheral scanning using ${ }^{125}$ I-tagged fibrinoge $\vec{n}$ (Flanc et al., 1968). In special clinical situations such as in a study of the natural history of deep vein thrombosis, or as a tool in measuring objectivelycthe

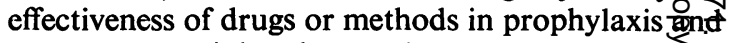
treatment, peripheral scanning represents an fing portant advance. However, as it is a method of dige nosing only peripheral deep vein thrombosis, the tecl: nique may have a limited role in clinical managemeng

The management of peripheral deep vein throms bosis requires therapeutic measures such as below the-knee compression and supportive bandaging leg elevation when resting, and a regime of activit and calf muscle exercises; these measures are de signed to limit venous stasis. It also implies a awareness by the clinician that clot may be forming elsewhere, particularly in the iliofemoral segment: Alerted to this possibility, the clinician is therefore. watchful for signs of iliofemoral involvement, ang understands that associated pulmonary embolis may have arisen from that segment. In the pas? failure to control pulmonary embolism by supers ficial femoral vein ligation below the profund termination has been due to clot formation in the iliofemoral segment. When it is confirmed that the peripheral segment is the only site of clot formatior superficial vein ligation below the profunda terminat tion is a satisfactory procedure in controlling embolism (Mavor \& Galloway, 1969a). At operzo tion, non-involvement of the iliofemoral segment should be confirmed radiographically.

\section{Iliofemoral (upper segment) venous thrombosis}

In iliofemoral venous thrombosis, clot formatio begins in the segment. The common iliac vein 
often initially involved, the left more so than the right in a ratio of $3: 1$. Thrombus formation may be non-occlusive without leg signs or occlusive with evidence of acute venous insufficiency. In the former pulmonary embolism is the presenting symptom, but eventual occlusion may result later, producing the 'late leg syndrome' (Mavor \& Galloway, 1967a). Pulmonary embolism complicates this discase in $50 \%$ of all cases, and is more common in right-sided involvement than left (Mavor \& Galloway, 1969a). It may be major and massive, and present day anticoagulant regimens are not adequately protective, mortality reaching $30 \%$ (Barker, 1959; Donaldson, Linton \& Rodkey, 1961).

In the presence of iliofemoral venous occlusion, the collateral arrangements are generally inadequate and acute venous insufficiency results. The early signs in such cases are discomfort and tenderness over the deep vein at the groin and in the suprainguinal region. Often calf tenderness and swelling may be present shortly before iliofemoral occlusion develops, and is undoubtedly in these circumstances due to venous stasis in and dilatation of the calf venous plexuses. As occlusion develops, there is an increase in the superficial venous pattern over the upper thigh and lower abdominal wall, and eventually swelling of the whole leg involving calf and thigh. When occlusion is sudden or extensive, the blue leg of phlegmasia caerulea dolens or even venous gangrene results. Without treatment designed to restore patency of the upper segment, the patient who survives will always have a degree of chronic venous insufficiency of the poor collateral circulation type with a swollen leg made worse by any position increasing venous stasis or circumstances increasing peripheral blood flow (Fig. 2). Such a leg represents another aspect of the postphlebitic syndrome (Cockett \& Jones, 1953; Mavor \& Galloway, 1967b), and may or may not be associated with lower leg skin induration or ulceration, which depends on perforator damage. In instances where the iliofemoral occlusion does not involve the whole segment, and particularly where direct channels such as venae comitantes and the ascending lumbar vein are present, the functional capability of the lower limb is better and morbidity less (Mavor \& Galloway, 1967b).

An aid to the diagnosis of iliofemoral venous thrombosis has been the recent introduction of the ultrasonic Doppler technique (Evans \& Cockett, 1969). This technique can be used as a guide and rough screening method in detecting iliofemoral thrombosis. It will be most accurate where thrombus is occlusive, and most inaccurate when thrombus is non-occlusive. Unfortunately, it is in the latter circumstances where there is non-occlusive thrombus in the iliofemoral segment, that patients are at most

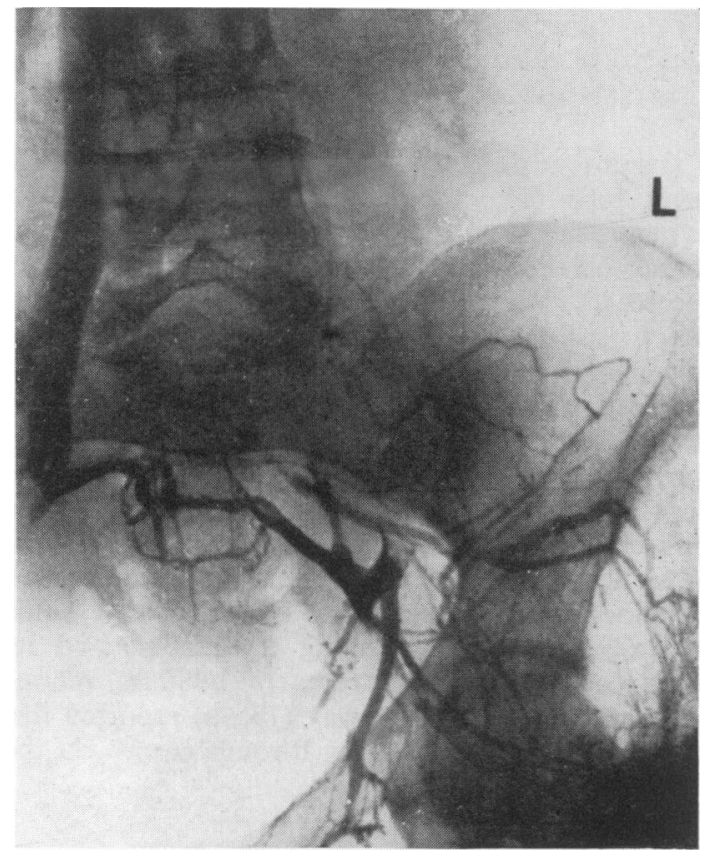

FIG. 2. Extensive left-sided iliofemoral segment occlusion. There is an extensive collateral network, the main venous drainage being to the contralateral internal iliac system and iliofemoral segment. Such cross-filling is usually associated with marked venous insufficiency in the affected limb.

risk from massive embolism. The method therefore cannot be relied upon to screen patients at risk from massive embolism, and cannot at the present time replace phlebography.

\section{Venous gangrene}

Where iliofemoral venous thrombosis is sudden and extensive, venous gangrene may arise. Unilateral venous gangrene is most apt to occur when the contralateral iliofemoral segment has been thrombosed previously, so that a collateral venous system via the contralateral internal iliac system is not possible. In other instances venous gangrene complicates bilateral iliofemoral and often caval involvement. Such severe and extensive occlusion leads to massive swelling of the legs which is progressive and leads in time to hypotension due to severe hypovolaemia due to blood loss into the limbs. Such a situation is not rectified by giving blood, which will only contribute to the swelling and clotting tendency. Because of the extensive intravenous clotting, there is also a sequestration of blood elements in the affected limbs. The utilization of platelets and their consequent reduction may lead to thrombocytopaenic purpura, usually in the affected limbs but also occasionally elsewhere in the 
body's subcutaneous tissues. Anticoagulants are dangerous in such a situation and may initiate or perpetuate tissue haemorrhage (Rosenberg \& Zullo, 1958).

The prognosis in venous gangrene is bad, mortality being high (Haimovici, 1950; Catchpole, 1957; Lowenthal \& May, 1965). Anticoagulant therapy has no beneficial effect on the course of the disease, and due to its risk should not be used. External iliac artery ligation may control hypotension and hypovolaemia and save life, usually at the expense of amputation. Clearance of the iliofemoral segment or the cava is imperative in such cases and should always be attempted. Although if undertaken late, clearance may be the exception rather than the rule in severe and fulminating cases of thrombo-embolism, an early and aggressive approach as regards venous thrombectomy is nevertheless indicated as it holds the only chance of arresting the disease and its complications. In seven cases of bilateral venous gangrene Mavor \& Galloway (1969a) reported five cases successfully treated by thrombectomy via the

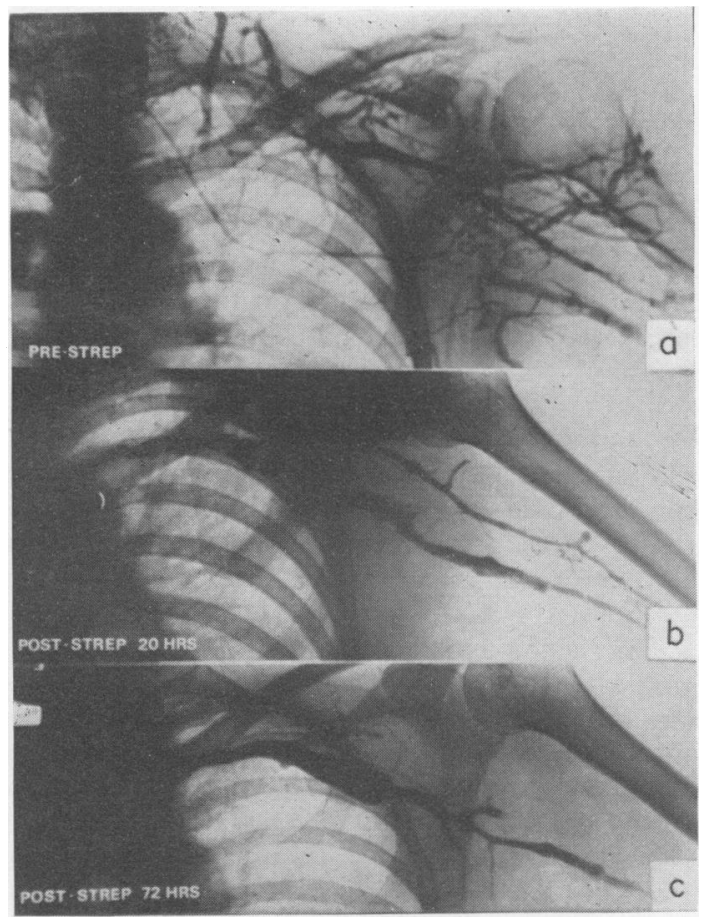

Fig. 3. This 50-year-old woman developed an extensive subclavian and innominate vein thrombosis after prolonged intravenous fluid therapy, for acute pancreatitis (a). Streptokinase was infused for $44 \mathrm{hr}$ through a catheter in the basilic vein. Marked lysis was produced by $20 \mathrm{hr}$ (b) and continued after streptokinase was discontinued (c). cava. All showed patency of the iliofemoral segments and cava at long-term follow-up.

\section{Subclavian vein thrombosis}

Subclavian vein thrombosis is uncommon. It ma站 be secondary to skeletal injury, to malignant disease such as bronchial carcinoma, to cardiac failure, of to surgery. Postoperative subclavian vein thrombosi has in the past complicated thoracoplasty for tuber culosis, and occasionally radical mastectomy. Morê often than is probably realized it may follow pros longed intravenous fluids delivered via long cephali $\vec{\odot}$ vein catheters (Fig. 3). Primary subclavian vein. thrombosis can occur in young adults. There may be a history of trivial injury. Embolism is uncommonge and the prognosis usually good because of the ade 3 quate collateral venous arrangements (Short, 1969) Because of the good prognosis, operative treatment is usually unnecessary, although in some cases where occlusion is extensive and pulmonary embolismo present, active therapy may be required (Fig. 3).

\section{Non-occlusive iliofemoral venous thrombosis}

Although it has been known for some time that clot formation in the iliofemoral segments may b* extensive without being occlusive (Servitt \& Gallag $\overrightarrow{0}$ her, 1961), it is only now being recognized clinicgly that these are the circumstances under which patient is at severe risk from pulmonary embolfing a risk gravely increased by events causing an increase in the negative phase of respiration. It is probable that in two-thirds of all patients who presenf with pulmonary embolism without leg signs, the source of the embolus is from non-occlusive clot ip the iliofemoral segment. It is in this context that femoral or intraosseous pertrochanteric venograph has been developed to visualize the iliofemoral segments, and plays such an important role in the management of pulmonary embolism, placing measure of control on an otherwise hypothetical clinical situation (Mavor \& Galloway, 1967a). Tho hazard from such an investigation is not great, bu the risk of pulmonary embolism is increased by badly conducted general anaesthesia (necessary with intraosseous injections) leading to over-breathing respiratory difficulties. In the presence of norf occlusive clot with pulmonary embolism, venous thrombectomy has given excellent results in produce ing complete clearance of the segment, therebof controlling embolism (Fig. 4) (Mavor \& Galloway 1969a).

\section{Occlusive iliofemoral venous thrombosis}

Iliofemoral venous occlusion represents the serious therapeutic challenge in deep vein thrombosis, and the problem of restoring upper segment patency is all important. Only by producing clearance and 


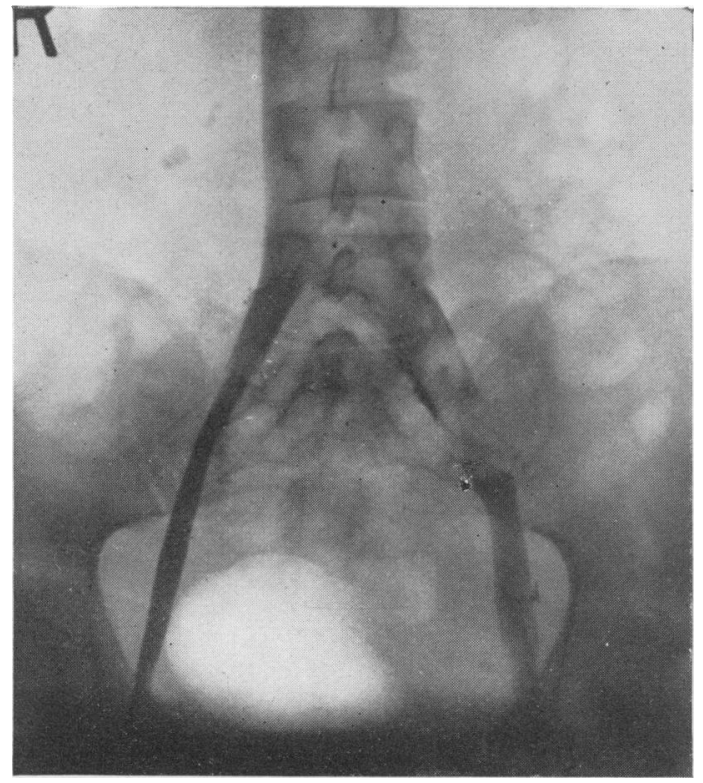

FIG. 4. Left non-occlusive iliofemoral thrombosis with thrombus mainly in the common iliac vein. As the main vein is not completely occluded, there is no collateral pattern.

maintaining patency of the segment will the risk of major embolism be removed and acute and chronic venous insufficiency of the poor collateral circulation type overcome.

\section{Venous thrombectomy}

The operation of venous thrombectomy without ligation or plication is the procedure of choice in most instances of iliofemoral venous occlusion. Whether or not embolism is present should not influence the decision to operate, but when embolism is present, preoperative phlebography to visualize the contralateral segment and inferior vena cava which may be the site of non-occlusive clot is required. Contra-indications to surgery are related to the patient's general condition, such as the presence of advanced malignancy, severe intercurrent disease, severe obesity, or to local factors such as groin infection or an already useless leg such as in hemiplegia.

The best results from thrombectomy will be obtained using radiographic operative control, balloon catheters and the saphenofemoral catheter technique for postoperative topical heparin (Fogarty \& Krippaehne, 1965; Mavor et al., 1969b). If necessary, postoperative phlebography can be carried out through the saphenofemoral catheter which is generally retained in position for 7 days (Mavor \& Galloway, 1967c). During surgery, it is not necessary to resort to methods of proximal control of the inferior vena cava, as without such control the incidence of embolism during surgery is well below 1\% (Mavor \& Galloway, 1969a).

As has been stated, complete clearance of the segment has been obtained in nearly $100 \%$ of cases in which the clot is not occlusive. Venographic studies have shown that in a series of 112 occlusive cases complete clearance has been obtained in $64 \%$ of all cases, and in $75 \%$ where surgery was undertaken within $48 \mathrm{hr}$ of the onset of complete occlusion (Fig. 5). In such instances embolism is controlled, and re-thrombosis either early or late, is unusual (Mavor \& Galloway, 1969a).

Incomplete clearance of the segment increases the incidence of these complications and detracts from the value of the procedure. The longer the delay after occlusion, the greater are the difficulties of clearance, and it is doubtful whether the operation is indicated $72 \mathrm{hr}$ or more after complete occlusion is clinically obvious, except in special circumstances. These relate to serious acute venous insufficiency with a 'blue leg' or even the more serious development of venous gangrene. Even partial clearance in such cases may diminish venous insufficiency by restoring function to important direct venous channels (Mavor \& Galloway, 1969a).

\section{Caval thrombectomy}

Venous thrombectomy via the inferior vena cava is indicated in severe insufficiency approaching gangrene, particularly if the iliofemoral occlusion is bilateral. Phlebographic evidence of clot in the cava particularly if associated with pulmonary embolism may also be an indication for opening the cava. In most instances thrombectomy via the common femoral vein will not have produced satisfactory clearance, and the need to open the cava will be dictated by the degree of venous insufficiency and the operative phlebographic findings. There is no doubt that in the problem of serious acute venous insufficiency, thrombectomy via a caval approach is worth more consideration (Mavor \& Galloway, 1969a).

\section{Caval ligation and plication}

It requires to be stressed that the aim of surgery in caval or iliofemoral venous thrombo-embolism is to restore patency by venous clearance, thereby controlling embolism and maintaining function. Interruption of the main venous channel proximal to the profunda termination is to be avoided if at all possible, and to use such methods widely in the presence of established thrombo-embolism is to court serious morbidity without necessarily controlling complications (DeMeester et al., 1967; Mavor \& Galloway, 1967a). However, in some long- 


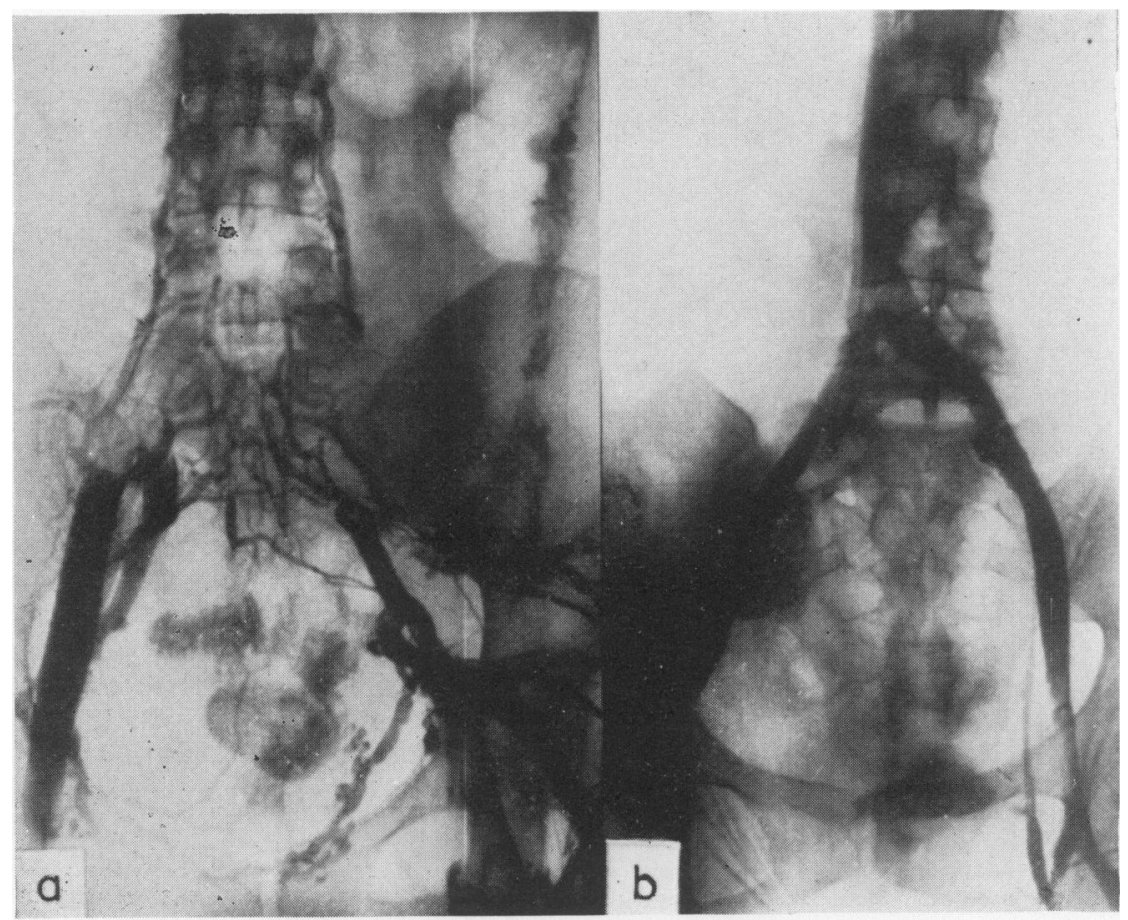

FIG. 5. This 26-year-old woman had been on oral contraceptive therapy (norlestrin) for 5 years. Thirteen days after vagal section and gastro-enterostomy, she complained of bilateral thigh discomfort and ankle swelling. Preoperative phlebography showed bilateraliliofemoral occlusion along with caval involvement (a). Bilateral venous thrombectomy produced complete clearance (b) (postoperative). Recovery was uneventful and 3 months later her legs were normal.

standing or recurrent cases of serious thromboembolism iliac or caval ligation may still be indicated in an endeavour to control embolism and save life. In such cases thrombectomy is unsatisfactory because of the duration of the disease. Following ligation in these circumstances there is a serious risk of venous gangrene and right heart failure, and even though ligation is proximal to all known clot, embolism may occur from fresh thrombus formed proximal to the point of ligation. In such cases there is little doubt that thrombolytic therapy if available is the better treatment.

\section{Management of pulmonary embolism}

It should always be remembered and the knowledge actively employed clinically that in iliofemoral thrombosis, whether non-occlusive or occlusive, embolism is common, while in peripheral thrombosis embolism is much less frequent and much less serious. It is certain that where embolism is the presenting symptom in the absence of leg signs, proper localization of the site of venous thrombosis is advantageous. As regards the peripheral segment, this is best achieved by the ${ }^{125}$ I-labelled fibrinogen

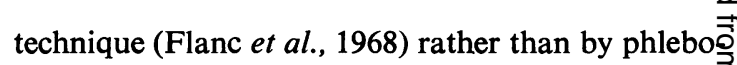
graphy, while in the more important iliofemora segment, phlebography is much more satisfactor (Mavor \& Galloway, 1969a). Present experience of such clinical methods suggests that in about two thirds of all cases there is definite evidence that the iliofemoral venous segment is involved. In most o these cases there is no peripheral activity (Mavor \& Galloway, 1969a). This confirms previous finding in retrospective studies (Mavor \& Galloway, 1967a) In the clinical management of pulmonary embolism the state of the iliofemoral segments should therefore always be known, and it is probably unnecessary to indulge in routine screening of the peripheral seger ments.

In the presence of established pulmonary emp bolism, the demonstration by phlebography that the iliofemoral segments are clear produces the assurance that major or fatal embolism is impossible. However it is still possible that the embolus has arisen frono the iliofemoral segment, and recurrent embolism leading to the need for repeated phlebography mas in due course reveal evidence of iliofemoral thrombusp

The presence of embolism complicating unilatera他 
iliofemoral occlusion is an indication for bilateral visualization of the iliofemoral segments prior to venous thrombectomy, so that non-occlusive thrombus in the contralateral segment or cava can be detected. Failure to do so may be the cause of recurrent or fatal postoperative embolism (Mavor et al., 1970a).

Lung scanning (Taplin et al., 1968) is of value in helping to establish the diagnosis of pulmonary embolism, especially where clinical signs, radiological and electrocardiographic findings are equivocal. The essential findings in early cases is an area of diminished vascularity in the pulmonary scan with a normal chest X-ray. In this way earlier and more certain diagnosis of pulmonary infarction is possible, allowing earlier assessment of the iliofemoral segments.

\section{Pulmonary embolectomy}

The operation of pulmonary embolectomy was first described by Trendelenberg in 1908, but successes reported in the literature have been few and far between. One of the difficulties encountered is that of accurate diagnosis. In massive embolism differentiation from severe myocardial infarction may be extremely difficult, and delay to carry out an emergency pulmonary angiogram may mean that in some instances the opportunity for pulmonary embolectomy is missed. However, in any centre with the available skills where immediate cardiac resuscitation is part of the routine treatment available for cardiac arrest, pulmonary embolectomy should always be considered as a line of treatment. Although recently streptokinase has been suggested as an alternative method of treatment, this drug is only of value if survival is to be measured in hours or days. Pulmonary embolectomy is to be preferred for the patient with massive embolism likely to die within the hour, and if it is being used for the right cases is probably best carried out without by-pass (Pisko-Dubienski, 1968). Streptokinase therapy and pulmonary embolectomy should never be combined because of the risk of uncontrollable haemorrhage. If the procedure of emboleztomy is successful it is then important to assess, perhaps by operative phlebography, the condition of the iliofemoral segments and cava, so that venous thrombectomy, when indicated, can be carried out without delay, and the risk of further embolism removed (Pisko-Dubienski, 1968).

\section{Mortality}

Mortality in iliofemoral venous thrombosis is usually due to pulmonary embolism. As previously stated, venous gangrene is known to carry a high mortality, but this fortunately is a rare condition. In a series of 302 cases of iliofemoral venous thrombosis treated by venous thrombectomy, the mortality from pulmonary embolism has been $4 \%$. Three deaths were considered as operative and nine deaths followed embolism covered a 3 months' postoperative period. In several of these, preoperative assessment by bilateral phlebography was not adequate, and embolism may have arisen from nonocclusive thrombus in the contralateral segment. Two of the nine deaths followed inferior vena cava ligation for recurrent embolism. In one other case, massive pulmonary embolism resulted during recovery from anaesthesia following phlebography, making a total of thirteen deaths in the saries of 302 (Mavor \& Galloway, 1969e).

\section{Oral contraceptives and venous thrombo-embolism}

It has been established that oral contraceptive agents with a high oestrogenic component produce a significant increase in the incidence of venous thrombo-embolism (British Medical Journal, 1967; Vessey \& Doll, 1968). However, the incidence is low and as these drugs are effective contraceptive agents, it has been estimated that in any given group the fatality rate is lower than results from 'escape pregnancies' in women using less reliable contracıptive methods used as a control series. However, further evidence must be produced to show that this is so, bearing in mind that there are now more precise ways of establishing the incidence of venous thrombo-embolism than counting fatalities.

How exactly oestrogenic compounds cause venous thrombosis is not as yet precisely known. Albrechtsen (1969) has shown that all patients under treatment develop an increased fibrinolytic activity in the blood, and suggests that those who are at risk are in a group who may fail to do so. This is in keeping with the work of Robertson (1969) who showed that in six out of seventeen women admitted with thrombo-embolism and taking oestrogen contraceptives, all six showed increased thrombocytic adhesiveness and diminished fibrinolytic activity. However, it is fair to state that as yet other studies have not supported these findings. During the state of simulated pregnancy produced by oestrogen therapy, iliofemoral phlebography shows that there is enlargement and congestion of the veins of the pelvis. It has been suggested that these changes if prolonged may lead to an increased vulnerability to thrombosis (Brodelius, Lorinc \& Nylander, 1969).

However, much more important from the clinician's point of view is the risk to patients on oestrogenic compounds who have to undergo surgical operations. Surgery itself is known to increase the incidence of deep vein thrombosis, and the same is true for blood transfusion and anaesthesia. It is also fair comment to say that many women on contraceptive therapy have had two or three children, and 
therefore are entering an age group when the need for elective surgery is increasing and when thromboembolic complications of the pill are greatest (Inman \& Vessey, 1968).

Recent experience suggests that serious postoperative venous thrombosis involving the iliofemoral segments and the inferior vena cava occurring in young women is most often in association with oral contraceptive drugs taken over a considerable time, and is not uncommon (Fig. 5) (Mavor \& Galloway, 1970c). This confirms the work of Nylander (1969) in Sweden, who found that of women under 35 years of age who developed postoperative iliofemoral venous thrombosis almost all had been on oral contraceptives. In a control group of young women developing less serious peripheral involvement none had been taking these agents (Nylander, 1969). There has too been a report of fatal mesenteric venous thrombosis in a young woman of 31 years (Lowry, Orr \& Wade, 1969).

These considerations give rise to considerable problems. It may be that there is a serious risk to young women being produced, which would not otherwise exist, and it may be that death from massive pulmonary embolism will soon be found to be on the increase in this group of postoperative patients. It is not known how soon after stopping these agents the risk of thrombo-embolism diminishes, and therefore to what extent these complications can be avoided by a policy of stopping therapy before undertaking elective surgical procedures. None-the-less with the present ignorance about this problem, it would appear prudent to take any reasonable measures and oral contraceptive drugs should probably be stopped 2 months before surgery. Where operation has to be undertaken as an emergency it must be recognized that these patients may represent a high risk group and prophylactic Dextran 70 and heparin should probably be given.

In the meantime it is to be hoped that further information on this important matter will be forthcoming at an early date.

\section{Thrombolytic therapy}

The use of thrombolytic agents is a further method of treatment in cases of intravenous thrombosis. Purified agents which can convert the inactive plasma globulin plasminogen, to the proteolytic enzyme, plasmin, are preferable to preparations of plasmin itself (Douglas \& McNicol, 1964). Of the two plasminogen activators available for thrombolytic therapy, streptokinase, produced from the $\beta$-haemolytic Streptococcus, has the disadvantage of being both pyrogenic and antigenic, but it is relatively cheap, whereas the alternative human product, urokinase, is neither toxic nor antigenic, but is so costly that its routine use is prohibited. It is also probable that as a thrombolytic agent streptokinase is more effective than urokinase (Mavor et al 1969c; Mavor et al., 1969d). Reports of streptokinase therapy continue to increase (Kakkar et al., 1969. Browse \& James, 1964).

Clinical and experimental experience suggests tha streptokinase is best given in high dosage into the region of the thrombus, rather than systemically (Mavor et al., 1969c; Karmody, 1969). Lysis wit clearance is readily produced in thrombus completelp occluding the iliofemoral segment, even with clot. known to be of 30 days' duration (Mavor et al 1969c). Administration of the drug is usually ter $\overrightarrow{\bar{\omega}}$ minated when lysis is complete or well advanced, og in rare instances when no lysis has occurred afte normally effective dosage. It is not exactly cleap. what laboratory tests are required during therapy but fibrinogen estimations are essential. If signifier cant hypofibrinogenaemia develops and a haemorr hagic state threatens, then the drug must be dis ${ }^{\omega}$ continued. Other reactions to the drug are neither frequent nor serious, and can be controlled by steroid administration during therapy (Mavor et al. $\rightarrow$ 1970a). Even though systemic thrombolytic activity is induced, this is no indication that lysis of thrombus is taking place, and objective evidence of throme bolysis is required for adequate dosage controlofit: is also known that after administration of the drigg is stopped, lysis may continue for up to 5 days (Mavo et al., 1969c). However, where there is prope objective control of therapy, best obtained in thष् iliofemoral segment by repeated phlebography via ad sapheno-femoral catheter, re-thrombosis, after strep tokinase therapy has been discontinued, can be recognized in about $25 \%$ of cases (Mavor et al. $\bar{D}$ 1969c). This occurs despite adequate and continuous heparin and warfarin sodium administration which should commence when streptokinase is discontinued There is no doubt that this aspect of streptokinasetherapy requires clarification. The mechanism of 3 . re-thrombosis is not as yet understood, and furtheri investigation of the clinical problem in the post- 3 streptokinase phase is necessary (Fig. 3).

Nevertheless, streptokinase is a drug of great promise in the effective therapy of venous thromboembolism. Although the susceptibility of thrombi ton lysis probably decreases with the age of the thrombus ${ }^{\text {G }}$ (Back et al., 1958), it is of interest and contrary to 0 expectation that in venous thrombo-embolism the age of the thrombus is not invariably critical ando that lysis of relatively old thrombi may be obtained 0 The incidence of re-thrombosis at the present time probably obviates the use of the drug in early cases? of iliofemoral thrombosis where venous thrombec- $\square$ tomy is so effective. Likewise it would seem un- $\bar{P}$ necessary to use streptokinase in peripheral venous thrombosis. At the present time the drug is the $\stackrel{\mathbb{Q}}{\Omega}$ 
treatment of choice when pulmonary embolism has produced serious cardio-respiratory failure as it has the capability both of relieving pulmonary arterial obstruction and preventing further embolism by removing its source (Browse \& James, 1964; Hirsh et al., 1967). It is just in such seriously ill patients with late or recurrent iliofemoral thrombo-embolism that venous surgery is precluded or is often, when undertaken so late, unavailing.

\section{References}

Albrechsten, O. (1969) Changes in coagulation and fibrinolysis during treatment with contraceptive pills. Societas Phlebologica Scandinavica. (In press.)

Alfors, K.E., Hint, H.C., Dhall, D.P. \& Matheson, N.A. (1968) Counteraction of platelet activity at sites of laserinduced endothelial trauma. British Medical Journal, 4, 430.

Allen, A.W. (1946) Venous thrombosis and pulmonary embolism. Surgery, Gynaecology and Obstetrics, 82, 232.

Aschoff, L. (1924) Lectures on Pathology, Chap. IX. Paul B. Hoeber, New York.

Back, N., Ambrus, J.L., Simpson, L. \& Shulman, S. (1958)) The study on the effect of streptokinase-activated plasmin on clots in various stages of organization. Journal of Clinical Investigation, 37, 864.

BARKER, W.F. (1959) The management of venous thrombosis and pulmonary embolism. Surgery, 45, 198.

BAUER, G. (1940) A venographic study of thrombo-embolic problems. Acta chirurgica Scandinavica, 84 Suppl. 61, 1.

Bennett, P.N., Dhall, D.P., McKenzie, F.N. \& Matheson, N.A. (1966) Effects of dextran infusion on the adhesiveness of human blood platelets. Lancet, ii, 1001 .

Blodgett, J.B. \& BeAtTie, E.J. (1946) Early postoperative rising. Surgery, Gynaecology and Obstetrics 82, 485.

British Medical Journal (1967) Preliminary risk of thromboembolic disease in women taking oral contraceptives. Communication to Medical Research Council, 2, 355.

Brodelius, A., Lorinc, P. \& Nylander, G. (1969) The $\mathrm{X}$-ray picture of P-pill thrombosis. Societas Phlebologica Scandinavica. (In press.)

Browse, N.L. (1968) Presentation to European Cardiovascular Society, London. July, 1968.

Browse, N.L. \& JAMES, D.C.O. (1964) Streptokinase and pulmonary embolism. Lancet, ii, 1039.

CAtChPole, B.N. (1957) Massive thrombophlebitis. Lancet, i, 343.

Cocketr, F.B. \& Jones, D.E.E. (1953) The ankle blow-out syndrome. Lancet, i, 17.

Cotron, L.T. \& ClARK, C. (1965) Anatomical localization of venous thrombosis. Annals of the Royal College of Surgeons, 36, 214.

Demeester, T.R., Rutherford, R.B., BlazeK, J.V. \& ZuIDEMA, G.D. (1967) Plication of the inferior vena cava for thromboembolism. Surgery, 62, 56.

Donaldson, G.A., Linton, R.R. \& Rodkey, D.V. (1961) A twenty-year survey of thrombo-embolism at the Massachusetts General Hospital, 1939-1959. New England Journal of Medicine, 265, 208.

Douglas, A.S. \& McNicol, G.P. (1964) Thrombolytic therapy. Proceedings of the Royal Society of Medicine, 57, 609.

Ehrich, W.E. \& KrumbhaAR, E.B. (1943) Frequent obstructive anomaly of mouth of left common iliac vein. American Heart Journal, $26,737$.

Evans, D.S. \& CocketT, F.B. (1969) Diagnosis of deep vein thrombosis with an ultrasonic Doppler technique. British Medical Journal, 2, 802.
Flanc, C., Kakkar, V.V. \& Clarke, M.B. (1968) The detection of venous thrombosis of the legs using ${ }^{125} \mathrm{I}-$ labelled fibrinogen. British Journal of Surgery, 55, 742.

FogARTY, T.J. \& KRIPPAEHNE, W.W. (1965) Catheter technique for venous thrombectomy. Surgery, Gynaecology and Obstetrics, 121, 362.

Frykholm, R. (1940) Pathogenesis and mechanical prophylaxis of venous thrombosis. Surgery, Gynaecology and Obstetrics, 71, 306.

Galloway, J.M.D. (1969) Ch.M. Thesis, Edinburgh.

GibBs, N.M. (1957) Venous thrombosis of the lower limb with particular reference to bed rest. British Journal of Surgery, 45, 209.

HaImovicI, H. (1950) Gangrene of the extremities of venous origin. Circulation, 1, 225.

Hellem, A.J. (1960) The adhesiveness of human blood platelets in vitro. Scandinavian Journal of Clinical and Laboratory Investigation, 12, Suppl. 51.

Hirsh, J., Hale, G.S., McDonald, I.G., McCarthy, R.A. \& CADE, J.F. (1967) Resolution of acute massive pulmonary embolism after pulmonary arterial infusion of streptokinase. Lancet, ii, 593.

Hobsley, M. \& PATEY, D.H. (1962) Muscular strain as a factor in post-operative thrombosis in the deep veins of the calf. British Journal of Surgery, 49, 620.

Homans, J. (1934) Thrombosis of the dep veins of the lower leg causing pulmonary embolism. New England Journal of Medicine, 211, 993.

INMAN W.H.W. \& VESSEY, M.P. (1968) Investigation of deaths from pulmonary, coronary, and cerebral thrombosis and embolism in women of child-bearing age. British Medical Journal, 2, 193.

Kakkar, V.V., Flanc, C., Howe, C.T., O'Shea, M. \& FluTe, P.T. (1969) Treatment of deep vein thrombosis. A trial of heparin, streptokinase and arvin. British Medical Journal, 1, 806.

Karmody, A.M. (1969) Ch.M. Thesis, Aberdeen.

Lambie, J.M., Barber, D.C., Dhall, D.P. \& Matheson, N.A. (1969) A controlled trial of venous thrombosis prophylaxis with dextran 70. British Journal of Surgery, 56, 619.

Lancet (1962) Correspondence. Unilateral idiopathic lymphoedema with obstruction of the common iliac vein by the common iliac artery, ii, 659.

Lowenthal, J. \& MaY, J. (1965) Phlegmasia caerulea dolens. British Journal of Surgery, 52, 584.

LOWRY, J.B., ORR, K.G. \& WADE, W.G. (1969) Infarction of the small intestine associated with oral contraceptives. Journal of the Irish Medical Association, 62, 260.

MCLachlin, A.D., McLachlaN, J.A., JoRY, T.A. \& RAWLING, E.G. (1960) Venous stasis in the lower extremities. Annals of Surgery, 152, 678.

Mclachlin, A.D. \& Paterson, J. (1951) Some basic observations on venous thrombosis and pulmonary embolism. Surgery, Gynaecology and Obstetrics, 93, 1.

McMurrich, J.P. (1908) Congenital adhesions in the common iliac vein. The Anatomical Record (1906-08), 1, 78.

Mavor, G.E. \& Galloway, J.M.D. (1967a) The iliofemoral segment as a source of pulmonary emboli. Lancet, $\mathbf{i}, 871$.

Mavor, G.E. \& Galloway, J.M.D. (1967b) Collaterals of. the deep venous circulation of the lower limb. Surgery, Gynaecology and Obstetrics, 125, 561.

Mavor, G.E. \& Galloway, J.M.D. (1967c) Radiographic control of iliofemoral venous thrombectomy. British Journal of Surgery, 54, 1019.

Mavor, G.E. \& Galloway, J.M.D. (1969a) Iliofemoral venous thrombosis. British Journal of Surgery, 56, 45.

Mavor, G.E., Galloway, J.M.D. \& MahafFy, R.G. (1969b) Venography in iliofemoral venous thrombus-embolism, Surgery, Gynaecology and Obstetrics, 129, 57. 
Mavor, G.E., Bennett, B., Galloway, J.M.D. \& Karmody, A.M. (1969c) Streptokinase in iliofemoral venous thrombosis. British Journal of Surgery, 56, 564.

Mavor, G.E., Ogston, D., Galloway, J.M.D. \& Karmcdy A.M. (1969d) Urokinase in iliofemoral venous thrombosis. British Journal of Surgery, 56, 571.

Mavor, G.E. \& Galloway, J.M.D. (1969e) Unpublished.

Mavor, G.E., Galloway, J.M.D. \& Karmody, A.M. (1970a) The surgical aspects of deep vein thromtosis. Proceedings of the Royal Society of Medicire, 63, 126.

Mavor, G.E. \& Galloway, J.M.D. (1970b) Prevention of rethrombosis following thrombolytic therapy. (In press.)

Mavor, G.E. \& Galloway, J.M.D. (1970c) Oral contraceptives and thrombo-embolism. Unpublished.

Murley, R.S. (1950) Postoperative venous thrombosis and pulmonary embolism with particular reference to current methods of treatment. Annals of the Royal College of Surgeons of England, 6, 283.

NylANDER, G. (1969) Meander-fenomenet i ytliga vener och dess pathogenetiska samband med varicer. Societas Phlebologica Scandinavica. (In press.)

Pisko-Dubienski, Z.A. (1968) A new approach to pulmonary embolism. British Journal of Surgery, 55, 138.

Robertson, B. (1969) Thrombolytic treatment of acute thrombosis in popliteal and femoral veins. Societas Phlebologica Scandinavica. (In press.)
RoSenberg, N. \& Zullo, R.J. (1958) Massive venous thrombosis associated with incoagulability of the bloo Archives of Surgery, 76, 981.

SEvitT, S. (1959) In: Modern Trends in Accident Surgery Medicine. (Ed. by R. C. Clarke, F. G. Badger and $S$ Sevitt), p. 247. Butterworth, London.

Sevitt, S. \& Gallagher, N.G. (1959) Prevention of venots thrombosis and pulmonary embolism in injured patient Lancet, ii, 981.

Sevitt, S. \& Gallagher, N.G. (1961) Venous thromtosis and pulmonary embolism. British Journal cf Surgery, 48 475.

SHORT, D.W. (1969) Subclavian vein thrombosis. Procee® ings of the Vascular Society of Great Britain and Ireland London, Novemter, 1969.

Taplin, G.V., Poe, N.D., Dore, E.K., Swanson, L.A Isawa, T. \& Greenderg, A. (1968) Proceedings of \&్ట్మ symposium, Salzburg, August, 1968. International Atom Energy Agency, Vienna, 1969.

TRENDELENBERG, F. (1908) Ueber die operative behandlun der embolie der lungenarterie. Archiv kliniscke Chirurgi Berlin, 86, 686.

VESSEY, M.P. \& Doll, R. (1968) Investigation of relatio between use of oral contraceptives and thromboembolic disease. British Medical Journal, 2, 199.

VIRCHOW, R. (1862) In: Gesammelte Adhardlungen zитे wissenchaftlichen Medizin, p. 458. Max Hirsch, Berlin. 